\title{
Fault Detection in Solar PV Systems Using Hypothesis Testing
}

\author{
${ }^{1}$ Fouzi Harrou, ${ }^{2,3}$ Bilal Taghezouit, Benamar Bouyeddou $4,5,{ }^{1}$ Ying Sun, ${ }^{2}$ Amar Hadj Arab \\ ${ }^{1}$ King Abdullah University of Science and Technology, CEMSE Division \\ Thuwal, 23955-6900, Saudi Arabia, fouzi.harrou@kaust.edu.sa \\ ${ }^{2}$ Centre de Développement des Energies, Renouvelables, CDER, 16340, Algiers, and Laboratoire \\ de Dispositif de Communication et de Conversion Photovoltaique, \\ ${ }^{3}$ Ecole Nationale Polytechnique Alger, 16200, Algeria. \\ ${ }^{4}$ Abou Bekr Belkaid University, STIC Lab., Department of Telecommunications, Tlemcen, Algeria \\ ${ }^{5}$ University of Saida-Dr Moulay Tahar, Department of Electronics, Faculty of Technology, Saida, Algeria
}

\begin{abstract}
The demand for solar energy has rapidly increased throughout the world in recent years. However, anomalies in photovoltaic (PV) plants can reduce performances and result in serious consequences. Developing reliable statistical approaches able to detect anomalies in PV plants is vital to improving the management of these plants. Here, we present a statistical approach for detecting anomalies in the DC part of PV plants and partial shading. Firstly, we model the monitored PV plant. Then, we employ a generalized likelihood ratio test, which is a powerful anomaly detection tool, to check the residuals from the model and reveal anomalies in the supervised PV array. The proposed strategy is illustrated via actual measurements from a 9.54 PV plant.
\end{abstract}

Index Terms-Photovoltaic systems, fault detection, partial shading, GLR test, statistical monitoring charts.

\section{INTRODUCTION}

Faced with the great energy transition in the world, the electricity generation using renewable energy has marked a fast-growing year-by-year. According to the International Renewable Energy Agency, a capacity of 171 gigawatts (GW) was added in 2018 primarily by new solar and wind, and this capacity additions represent $84 \%$ of all new capacity installed in 2018. This brings total renewable energy generation capacity up to $2,351 \mathrm{GW}$ at the end of 2018 .

Failures in PV arrays are frequently challenging to avoid and may generate a loss of energy, shutdowns or even in critical safety problems [1]-[3] as illustrated in Figure 1. As an example of critical failures in PV plants, Figure 1 are taken respectively from two PV plants that were burned in the United States (Bakersfield, CA, and Mount Holly, NC) in 2009 and 2011, respectively [1] due to undetected anomalies in their DC parts. Thus, continuous and accurate monitoring of PV plants is crucial for the benefit of the PV project investments and to reach the expected energy yield and maximize the PV system's useful life. Indeed, a monitoring system is an indispensable part to maintain optimal performance of PV systems, which allows to increase reliability, efficiency, service lifetime performance and safety in PV plants and to reduce the cost of operation and maintenance [4].
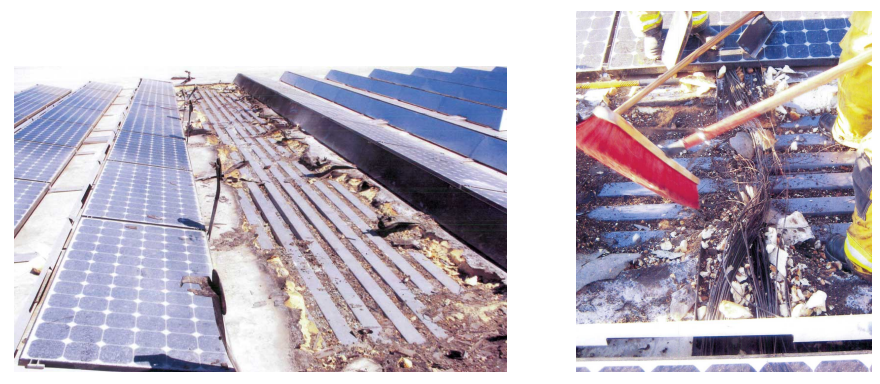

Fig. 1. Left: $383 \mathrm{kWp}$ PV plant, California (fire hazard, 2009). Right: 1,208 MWp PV plant, NC (fire hazard, 2011) [1].

Accurate and early detection of faults in PV systems is becoming the backbone to facilitate developing efficient PV systems that meet the desired requirements and specifications [4], [5]. All over the years, various data-based and modelbased methods have been introduced to improve the detection of faults in PV plants [3], [6]. For instance, In [7], a detection procedure based on a double exponential chart is proposed to monitor photovoltaic systems. In [8], an approach to monitor in an automatic way the degradation of the PV system by analyzing the I-V features is introduced. Indeed, analyzing I-V characteristics for several values of irradiance and temperature by comparing the measured I-V with the simulated one are frequently used in the literature to detect and identify the failure appearing in the PV module, string or array (e.g., shading, short circuit, aging and degradation, and the dust effect) [9]. In [10], the authors applied a neural network algorithm for fault detection using I-V characteristics. However, it should be noted that the use of I-V characteristics requires switching off the PV generator to collect measurements of the I-V characteristic, which limits the application of this approach for online monitoring. In [11], a method coupling criteria in the time and frequency domain is introduced to detect parallel arc faults in the DC side of PV arrays. In [12], an approach based on a wavelet transform using the current at the exit of a PV panel is suggested to detect DC side parallel arc fault. In [13], authors propose a classifier to identify faults and shadows that occurred in PV based on two 
new indicators related to radiation and ambient temperature. In [14], an approach has been developed for fault detection and localization if PV systems by merging the Gaussian kernelFuzzy $\mathrm{C}$ Means algorithm for unsupervised clustering and a probabilistic neural network to model fault process. In [15], an unsupervised strategy is introduced by using the one-diode model and one-class SVM [15]. In one-class SVM, using faultfree data the optimal decision hyperplane is determined to separate normal measurements from faulty data, and then used for fault detection. The key characteristics of this approach are its flexibility to handle nonlinear and non-Gaussian data, and it is assumption-free on data. In [16], several kernel-based machine learning methods have been adopted for photovoltaic plant monitoring. Many other machine learning algorithms have been widely exploited in the diagnosis of photovoltaic systems including random forest [17], decision tree algorithm [18], and extreme learning machine [19].

Statistical process control methods are considered as a powerful tool for monitoring production systems. They are widely used in several fields including manufacturing [20], healthcare [21], and traffic management [22]. Numerous univariate anomaly detection methods were designed in the literature such as the cumulative sum schemes, and the exponential smoothing methods. However, these monitoring approaches are generally designed so that they are more or less sensitive to a specific range of changes [23]. For instance, Shewhart-type charts are more suited to detect large changes, whereas other charts are more appropriate to uncover small changes [4]. In fact, CUSUM and EWMA methods may be set to be very suitable for sensing small changes, but they lack efficiency in detecting large changes. To cope with this challenge, one approach to get a desirable detection performance for different ranges of changes is to employ the monitoring scheme based on a likelihood ratio test (GLR scheme) [24], [25]. The GLR has demonstrated good ability in detecting a wide range of settings making them advantageous for use in real applications [25]. A desirable characteristic of GLR schemes is their flexibility and ease to design; they need only the detection limit to be defined [25], [26]. In recent years, deep learning-based methods have received considerable attention in the diagnosis of PV systems because of their extended performance and ability to learn complex features. Several deep learning algorithms have been applied to improve the monitoring of PV systems, such as a convolutional neural network [27], long short term machine [28], and showed superior performance compared to the shallow learning methods.

The objective of this work is to propose an accurate anomaly detector for supervising the DC part of a PV plant. To do so, we proposed an efficient statistical scheme using a generalized likelihood ratio (GLR) test. GLR detector, which is a statistical hypothesis testing approach, provides desirable detection capability via maximization of the detection probability of anomalies for predefined false alarm probability. It has been widely exploited in several industrial applications. Particularly, this approach benefits from the good ability of the GLR chart to sense the deviations between normal and abnormal features to better detect faults in PV systems. It should be pointed out that univariate anomaly detection schemes (e.g., GLR) are designed based on the hypothesis that data are uncorrelated. However, measurements from the DC part of PV plants are autocorrelated. To remedy this difficulty, the proposed detection framework amalgamates a simulation model and a GLR test to detect anomalies in PV plants. First, a simulation model is used for describing the evolution of MPP variables by using as input the ambient temperature and tilted solar irradiance measurements. Then, the GLR is used for checking the residuals generated from the simulation model to uncover anomalies in PV plants. The capacity of the proposed scheme is assessed via practical data from a $9.54 \mathrm{kWp} \mathrm{PV}$ array.

The following section describes the considered PV array. Then, Section III presents the simulation model. In Section IV, the designed anomaly detection methodology is presented. Section V checks the detection performance of the developed procedure. Lastly, Section VI offers some concluding remarks of this study.

\section{PV SYSTEM DSCRIPTION}

The fault detection method proposed in this study is assessed using measured data gathered from a grid-tied PV system in Algiers. The PV array consists of 90 modules (Isofotron 106/12) with a total peak dc power of $9.54 \mathrm{KWp}$. The PV plant is formed of a PV array of 90 panels. The PV system is formed with three similar PV generators of 3.18 $\mathrm{kWp}$.

Every PV generator is linked in a way to have the following configuration: two parallel string of fifteen PV panels in series. The PV generator is cabled through an electrical protection cabinet to the inverter (Fronius IG 30). The essential electrical features of the PV generator and inverter are given in Table I and Table II, respectively.

TABLE I

ESSENTIAL PROPERTIES OF PV PANEL AND GENERATOR.

\begin{tabular}{lccccc}
\hline Parameters & VoC$_{\text {OV }}$ & $\mathbf{I}_{\text {SC }}(\mathbf{A})$ & $\mathbf{V}_{\text {MPP }}(\mathbf{V})$ & $\mathbf{I}_{\mathbf{M P P}}(\mathbf{A})$ & $\mathbf{P}_{\mathrm{MPP}}(\mathbf{W})$ \\
\hline PV Module & 21.6 & 6.54 & 17.4 & 6.1 & 106 \\
\hline PV Generator & 324 & 13.08 & 261 & 12.2 & 3180 \\
\hline
\end{tabular}

TABLE II

ESSENTIAL PROPERTIES OF THE USED PV INVERTER.

\begin{tabular}{lccccc}
\hline Parameters & $\begin{array}{c}\mathbf{P}_{\mathrm{AC}} \\
\text { Nominal }\end{array}$ & $\begin{array}{c}\mathbf{V}_{\mathrm{MPP}} \\
\text { range }\end{array}$ & $\begin{array}{c}\text { Efficiency } \\
\text { range }\end{array}$ & $\begin{array}{c}\mathbf{V}_{\mathbf{A C}} \\
\text { range }\end{array}$ & $\begin{array}{c}\text { Frequency } \\
\text { range }\end{array}$ \\
\hline \multirow{2}{*}{ Value } & 2500 & $150-400$ & $92.7-94.3$ & $195-253$ & $49.8-50.2$ \\
& $\mathrm{~W}$ & $\mathrm{~V}$ & $\%$ & $\mathrm{~V}$ & $\mathrm{~Hz}$ \\
\hline
\end{tabular}

Figure 2 displays the monitoring system used for requiring and analyzing the collected measurements using the sensors. Table III summarizes the features of the sensors utilized to measure electrical and meteorological data from the PV array. 


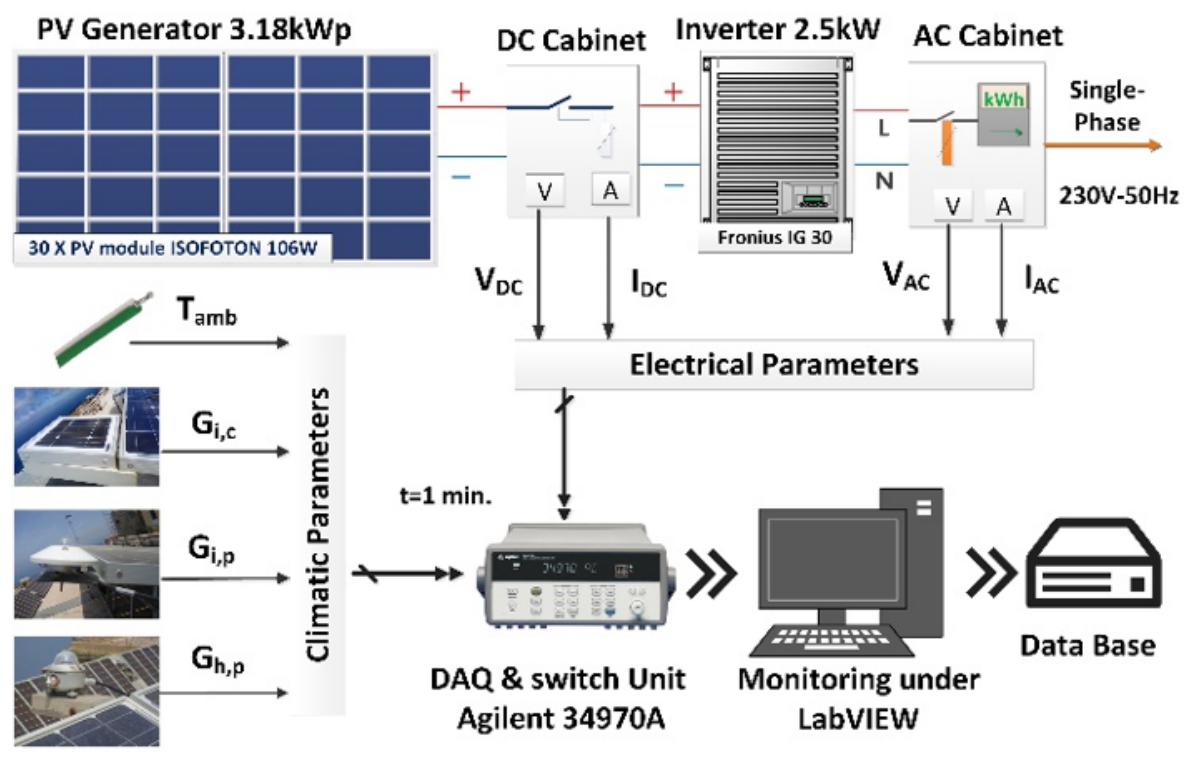

Fig. 2. Block chart of one PV sub-system.

TABLE III

ESSENTIAL PROPERTIES OF THE USED MONITORING SENSORS.

\begin{tabular}{|c|c|c|c|c|c|}
\hline \multicolumn{3}{|c|}{ Measured parameters } & Symbol & Sensor type & $\begin{array}{c}\text { Sensor } \\
\text { Reference }\end{array}$ \\
\hline \multirow{4}{*}{ Climatic } & Temperature & Ambient & $T_{a m b}$ & $\begin{array}{c}\text { Thermocouple } \\
\text { K }\end{array}$ & - \\
\hline & \multirow{3}{*}{$\begin{array}{c}\text { Global } \\
\text { Irradiance }\end{array}$} & \multirow{2}{*}{ Tilted_27 $7^{\circ}$} & $G_{I, C}$ & $\begin{array}{c}\text { PV reference } \\
\text { cell }\end{array}$ & Isofoton \\
\hline & & & $G_{l, p}$ & \multirow{2}{*}{ Pyranometer } & \multirow{2}{*}{$\begin{array}{c}\text { Kipp \& } \\
\text { zonen } \\
\text { CM } 11\end{array}$} \\
\hline & & Horizontal & $G_{H, p}$ & & \\
\hline \multirow{4}{*}{ Electrical } & \multirow{2}{*}{ Voltage } & DC & $V_{D C}$ & $\begin{array}{l}\text { Voltage } \\
\text { divider }\end{array}$ & - \\
\hline & & $\mathrm{AC}$ & $V_{A C}$ & $\begin{array}{c}\text { Voltage } \\
\text { transformer }\end{array}$ & - \\
\hline & \multirow{2}{*}{ Current } & $\mathrm{DC}$ & $I_{D C}$ & \multirow{2}{*}{$\begin{array}{l}\text { Hall Effect } \\
\text { Closed-loop }\end{array}$} & \multirow{2}{*}{$\begin{array}{l}\text { F.W. BELL } \\
\text { CLSM-50S }\end{array}$} \\
\hline & & $\mathrm{AC}$ & $I_{A C}$ & & \\
\hline
\end{tabular}

This study focuses on designing a monitoring chart for uncovering anomalies in the DC part of a PV plant through the use of tilted irradiance and ambient temperature as input variables.

\section{PV ARRAY MODELING}

A PV solar cell is usually modeled using a single diode model (SDM) (Figure 3), and the equation representing the $\mathrm{I}-\mathrm{V}$ characteristics is [29]

$$
I=I_{p h}-\underbrace{I_{0}\left[\exp \left(\frac{q\left(V+R_{s} I\right)}{n k_{B} T}\right)-1\right]}_{I_{d}}-\underbrace{\frac{V+R_{s} I}{R_{s h}}}_{I_{s h}},
$$

where $I_{0}$ refers to the dark saturation current, $q$ denotes the electronic charge, $k_{B}$ refers to the constant of Boltzmann and $n$ denotes the diode's ideality factor. There are fine five parameters with unknown values in Equation (1), $I_{p h}, I_{0}, n$,
$R_{s}$ and $R_{s h}$, which have a core role in in the prediction quality of the SDM.

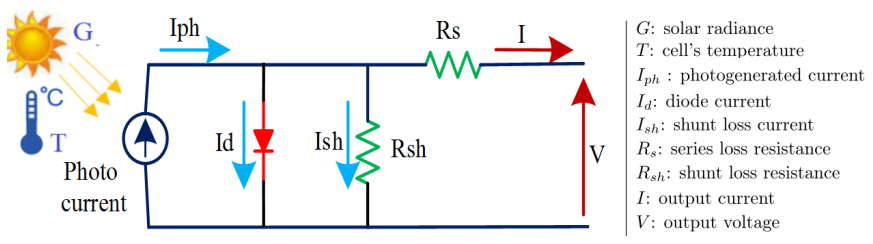

Fig. 3. Representation of SDM representing a PV cell.

Generally speaking, the designed anomaly detection procedure is accomplished by doing the next steps: (1) compute the parameters of SDM, (2) build and test a simulation model of the entire PV system using PSIM $^{T M} /$ Matlab $^{T M}$ co-simulation [30], and lastly evaluate residuals using the GLR to uncover abnormal condition in the PV system under monitoring.

\section{GLR-BASED FAULT DETECTOR FOR PV SYSTEM MONITORING}

Here, the GLR detector and its application in monitoring PV systems are briefed.

\section{A. GLR approach}

The GLR test is an efficient statistical detector to decide between two composite hypotheses. It has been widely applied to anomaly detection in time-series data in different. applications, including air quality [25], strain detection in hospitals [16], traffic congestion detection [26], and train positioning [24]. Importantly, a GLR-based chart can separate the null hypothesis $\mathcal{H}_{0}$ from the alternative one $\mathcal{H}_{1}$ based on only measured data. Let us consider the vector $Y=\left[y_{1}, y_{2}, \ldots, y_{n}\right] \in \mathbb{R}^{n}$ is created using one of the following Gaussian distributions: 


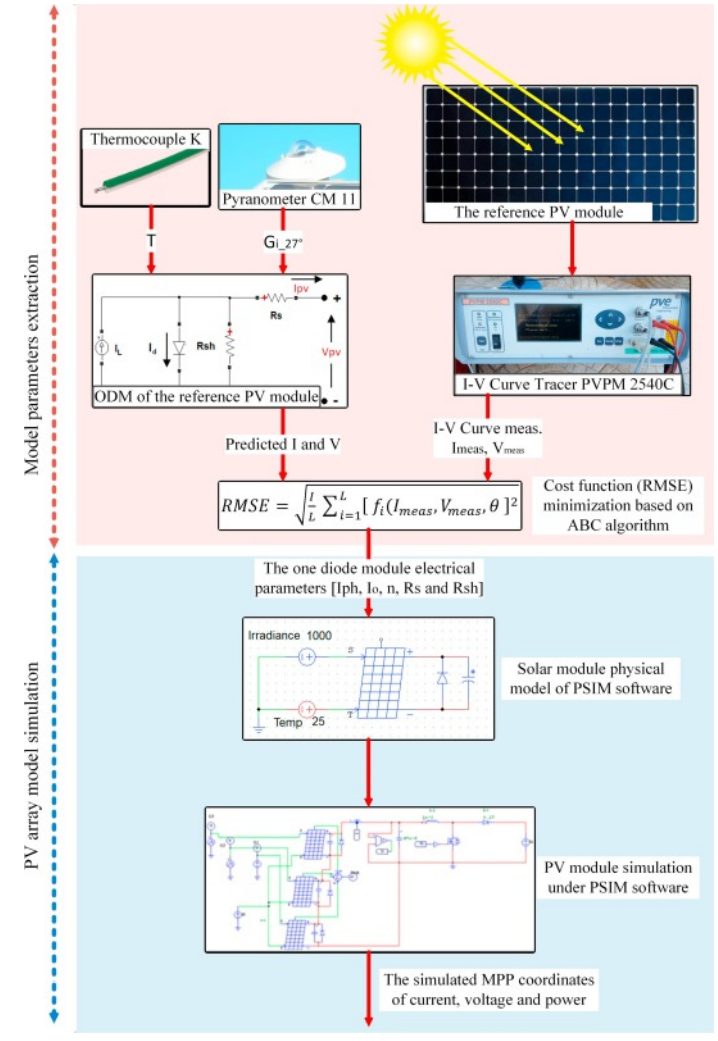

Fig. 4. Principal steps to model a PV plant [30].

$$
\left\{\begin{array}{l}
\mathcal{H}_{0}=\left\{Y \sim \mathcal{N}\left(0, \sigma^{2} I_{n}\right)\right\} \\
\mathcal{H}_{1}=\left\{Y \sim \mathcal{N}\left(\theta \neq 0, \sigma^{2} I_{n}\right)\right\}
\end{array}\right.
$$

Here, $\theta$ denotes the anomaly level, and $\sigma^{2}>0$ denotes the variance.

Here, $\mathcal{H}_{0}$ denotes the null hypothesis that represents the anomaly-free situation, and the alternative hypothesis, $\mathcal{H}_{1}$, describes the faulty case. The GLR scheme separates between $\mathcal{H}_{0}$ and $\mathcal{H}_{1}$ based on the comparison of $\mathcal{L}(Y)$ to the control limit, $h(\alpha)$.

$$
\delta(Y)=\left\{\begin{array}{l}
\mathcal{H}_{0} \text { if } \mathcal{L}(Y)=2 \log \frac{\sup _{\theta \in \mathbb{R}^{n}} f_{\theta}(Y)}{f_{\theta=0}(Y)}<h(\alpha) \\
\mathcal{H}_{1} \text { else. }
\end{array}\right.
$$

Consequently, the GLR detection statistic, $\mathcal{L}(Y)$, is defined as

$$
\mathcal{L}(Y)=2 \log \sup _{\theta}\left\{\exp \left\{-\frac{\|Y-\theta\|_{2}^{2}}{2 \sigma^{2}}\right\} / \exp \left\{-\frac{\|Y\|_{2}^{2}}{2 \sigma^{2}}\right\}\right\},
$$

where $f_{\theta}(Y)=\frac{1}{(2 \pi)^{\frac{n}{2}} \sigma^{n}} \exp \left\{-\frac{1}{2 \sigma^{2}}\|Y-\theta\|_{2}^{2}\right\}$ is the pdf of $Y$. Then, (3) can be expressed as

$$
\begin{aligned}
\mathcal{L}(Y) & =\frac{1}{\sigma^{2}}\left\{\min _{\theta}\|Y-\theta\|_{2}^{2}+\|Y\|_{2}^{2}\right\} \\
& =\frac{1}{\sigma^{2}}\left\{\|Y-\widehat{\theta}\|_{2}^{2}+\|Y\|_{2}^{2}\right\} .
\end{aligned}
$$

The estimation of $\theta$ is expressed as $\widehat{\theta}=\arg \min _{\theta}\|Y-\theta\|_{2}^{2}=Y$. Concerning the likelihood ratio, $\mathcal{L}(Y)$, it is re-written as,

$$
\mathcal{L}(Y)=\frac{1}{\sigma^{2}}\left\{\|Y\|_{2}^{2}\right\} \text {. }
$$

It is worth noticing that the detection threshold $h(\alpha)$, is defined such that the desired probability of false alarms is a priori fixed.

$$
\mathbb{P}_{0}(\mathcal{L}(Y) \geq h(\alpha))=\alpha .
$$

Overall, if the GLR statistic is within the control limit, then the supervised PV array is operating normally; otherwise, there is a potential anomaly.

\section{B. Monitoring PV systems using GLR-based approach}

Anomaly detection based on the GLR approach is realized into three stages as summarized in Figure 5. First, the model of the PV array is built using the training. Here, the ABC method is utilized to find the optimized electrical parameters of the SDM. Second, for testing unseen data, the build model is applied for estimating the MPP power, current, and voltage using measured temperature and irradiance. Residuals, which are the dissimilarity between predicted values of MPP variables from the model and the measured ones, are used to detect anomalies. Finally, the efficiency of the PV array is checked by applying the GLR scheme on the residuals from the model (Figure 5).

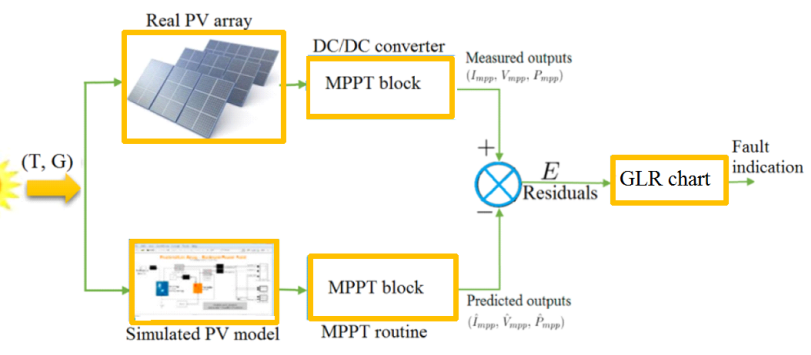

Fig. 5. Diagram of the proposed monitoring procedure.

\section{RESULTS AND DISCUSSION}

Now, the proposed anomaly detection approach will be applied to actual data given in Section II.

Firstly, by using the best-so-far ABC optimization algorithm we identify the parameters of the PV module based on the (I-V) curve measurements (Table IV). Next, we used the computed parameters for predicting (I-V) curve via the SDM (Eq. (1)).

TABLE IV

ESTIMATED PARAMETERS OF THE SDM.

\begin{tabular}{l|l|l|l|l|l|l}
\hline & $I_{p h}[\mathrm{~A}]$ & $I_{0}[\mathrm{~A}]$ & $n$ & $R_{s}[\Omega]$ & $R_{s h}[\Omega]$ & $\mathrm{RMSE}$ \\
\hline ISOFOTON 106-12 & 6.54 & $1.11 \mathrm{e}-0.5$ & 1.66 & 0.1474 & 202.6 & 0.014 \\
\hline
\end{tabular}

The built model is tested based on three-day profiles of temperature and irradiance as input to the model. Figure 6 indicates that peak power measurements $\left(\mathrm{Pmpp}_{\text {meas }}\right)$ fit with the estimated one $\left(\mathrm{Pmpp}_{\text {sim }}\right)$. 


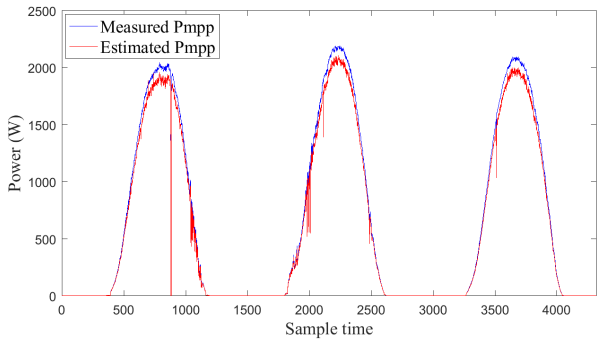

Fig. 6. Gathered and predicted MPP power.

\section{A. Detection results}

Herein, four types of scenarios are considered to evaluate the abilities of the GLR-based approach: two short-circuit faults, an open-circuit fault, and four PV modules temporary shaded (Figure 7).

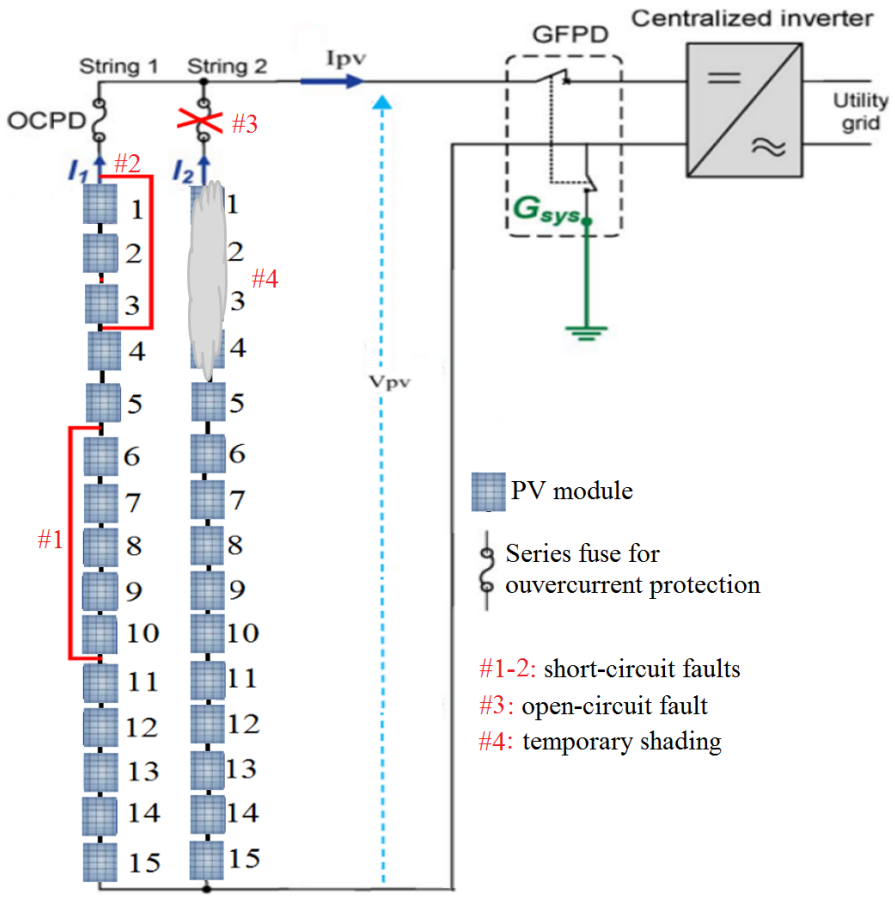

Fig. 7. Four failures tested in this study.

1) Short circuit faults: The results of the GLR approach based MPP power residuals when a short-circuit fault in five modules occurred are demonstrated in Figure 8. As expected, one can see that the GLR statistic exceeded the control limit indicating the presence of an anomaly.

Also, the performance of the proposed approach is verified when short-circuit fault occurred in three modules as shown in Figure 7 (Fault \#2). The monitoring result in Figure 9 and clearly confirms the capacity of the GLR approach to detect this short-circuit fault.

2) Open circuit fault: This scenario aims to test the GLR chart in the case of open-circuit faults (see fault \#4 in Figure 7) that happened from time point 300 to 400. The detection results are displayed in Figure 10. As shown in Figure 10, the GLR approach detect successfully this fault.

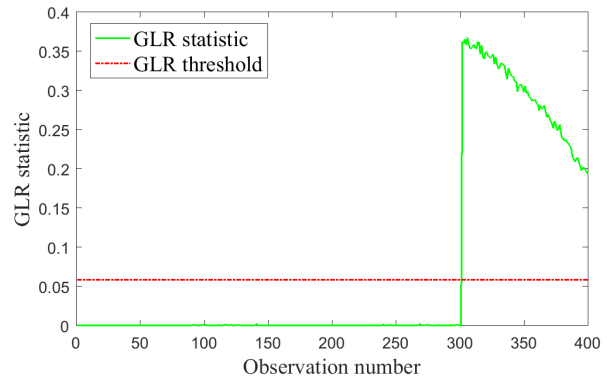

Fig. 8. Result of the GLR approach in the case of Fault \#1.

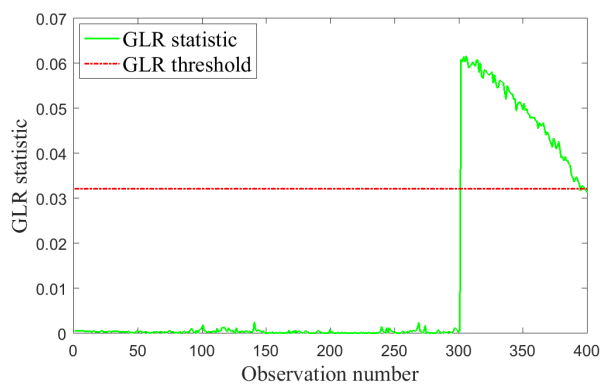

Fig. 9. Result of the GLR approach in the case of Fault \#2.

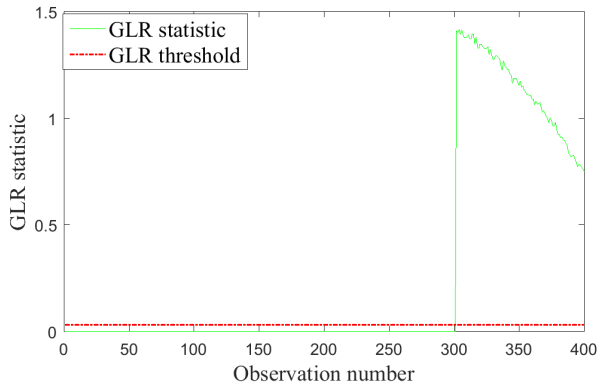

Fig. 10. Result of the GLR approach in the case of Fault \#3.

3) Example with temporary shading: In this scenario, we test the capability of the GLR monitoring scheme when temporary shading happened (Figure $7, \# 4$ ) in the time interval from 150 to 250 . Figure 11 shows the detection results of the GLR test. This result confirms the capacity of the GLR-based approach to detect temporary shading.

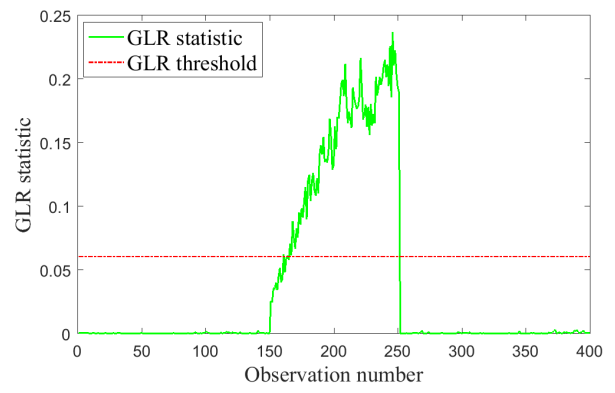

Fig. 11. Result of the GLR approach in the case of Fault \#4. 


\section{CONCLUSION}

In practice, photovoltaic plants are frequently exposed to anomalies that can reduce their productivity and safety. The present work deals with the fault detection problem in the DC side of PV systems using a model-based approach. Basically, the proposed detection scheme admits two stages: first, a reference simulation model is constructed for mimicking the nominal evolution of the inspected PV array, and it is used for residuals generation to uncover potential faults. After that, the GLR scheme is implemented to verify the residuals for uncovering potential faults. Successful detection results have been achieved when using databases generated from a 9.54 Kwp PV system. The results testify the sensitivity of the GLRbased scheme in detecting short-circuit, open-circuit faults, and temporary shading.

\section{ACKNOWLEDGEMENT}

This publication is based upon work supported by King Abdullah University of Science and Technology (KAUST), Office of Sponsored Research (OSR) under Award No: OSR2019-CRG7-3800. This publication is validated by experimental data produced within the photovoltaic solar energy division of the Centre de Développement des Energies Renouvelables (CDER).

\section{REFERENCES}

[1] B. Brooks. The bakersfield fire: A lesson in ground-fault protection. SolarPro, 4(2), 2011.

[2] Mohammed Khorshed Alam, Faisal Khan, Jay Johnson, and Jack Flicker. A comprehensive review of catastrophic faults in PV arrays: types, detection, and mitigation techniques. IEEE Journal of Photovoltaics, 5(3):982-997, 2015.

[3] Dhanup S Pillai, Frede Blaabjerg, and Natarajan Rajasekar. A comparative evaluation of advanced fault detection approaches for pv systems. IEEE Journal of Photovoltaics, 9(2):513-527, 2019.

[4] Elyes Garoudja, Fouzi Harrou, Ying Sun, Kamel Kara, Aissa Chouder, and Santiago Silvestre. Statistical fault detection in photovoltaic systems. Solar Energy, 150:485-499, 2017.

[5] Bilal Taghezouit, Fouzi Harrou, Ying Sun, Amar Hadj Arab, and Cherif Larbes. Multivariate statistical monitoring of photovoltaic plant operation. Energy Conversion and Management, 205:112317, 2020.

[6] Siva Ramakrishna Madeti and SN Singh. A comprehensive study on different types of faults and detection techniques for solar photovoltaic system. Solar Energy, 158:161-185, 2017.

[7] Bilal Taghezouit, Fouzi Harrou, Ying Sun, Amar Hadj Arab, and Cherif Larbes. A simple and effective detection strategy using double exponential scheme for photovoltaic systems monitoring. Solar Energy, 214:337-354, 2021.

[8] Masaki Miwa, Sanshiro Yamanaka, Hajime Kawamura, Hideyuki Ohno, and Hideaki Kawamura. Diagnosis of a power output lowering of pv array with a (-di/dv)-v characteristic. In 2006 IEEE 4th World Conference on Photovoltaic Energy Conference, volume 2, pages 24422445. IEEE, 2006.

[9] Yihua Hu, Bin Gao, Xueguan Song, Gui Yun Tian, Kongjing Li, and Xiangning He. Photovoltaic fault detection using a parameter based model. Solar Energy, 96:96-102, 2013.

[10] W Chine, A Mellit, Vanni Lughi, A Malek, Giorgio Sulligoi, and A Massi Pavan. A novel fault diagnosis technique for photovoltaic systems based on artificial neural networks. Renewable Energy, 90:501512, 2016.

[11] Chuxuan He, Longhua Mu, and Yijian Wang. The detection of parallel arc fault in photovoltaic systems based on a mixed criterion. IEEE Journal of Photovoltaics, 7(6):1717-1724, 2017.
[12] Silei Chen, Xingwen Li, and Jiayu Xiong. Series arc fault identification for photovoltaic system based on time-domain and time-frequencydomain analysis. IEEE Journal of Photovoltaics, 7(4):1105-1114, 2017.

[13] R Hariharan, M Chakkarapani, G Saravana Ilango, and C Nagamani. A method to detect photovoltaic array faults and partial shading in pv systems. IEEE Journal of Photovoltaics, 6(5):1278-1285, 2016.

[14] Honglu Zhu, Lingxing Lu, Jianxi Yao, Songyuan Dai, and Yang Hu. Fault diagnosis approach for photovoltaic arrays based on unsupervised sample clustering and probabilistic neural network model. Solar Energy, 176:395-405, 2018.

[15] Fouzi Harrou, Abdelkader Dairi, Bilal Taghezouit, and Ying Sun. An unsupervised monitoring procedure for detecting anomalies in photovoltaic systems using a one-class support vector machine. Solar Energy, 179:48-58, 2019.

[16] Fouzi Harrou, Farid Kadri, Ying Sun, and Sofiane Khadraoui. Monitoring patient flow in a hospital emergency department: Armabased nonparametric glrt scheme. Health Informatics Journal, 27(2):14604582211021649, 2021.

[17] Zhicong Chen, Fuchang Han, Lijun Wu, Jinling Yu, Shuying Cheng, Peijie Lin, and Huihuang Chen. Random forest based intelligent fault diagnosis for PV arrays using array voltage and string currents. Energy Conversion and Management, 178:250-264, 2018.

[18] Rabah Benkercha and Samir Moulahoum. Fault detection and diagnosis based on c4. 5 decision tree algorithm for grid connected pv system. Solar Energy, 173:610-634, 2018.

[19] Zhicong Chen, Lijun Wu, Shuying Cheng, Peijie Lin, Yue Wu, and Wencheng Lin. Intelligent fault diagnosis of photovoltaic arrays based on optimized kernel extreme learning machine and iv characteristics. Applied energy, 204:912-931, 2017.

[20] Jose A Romagnoli and Ahmet Palazoglu. Introduction to process control. CRC press, 2005.

[21] Farid Kadri, Fouzi Harrou, Sondès Chaabane, Ying Sun, and Christian Tahon. Seasonal ARMA-based SPC charts for anomaly detection: Application to emergency department systems. Neurocomputing, 173:2102$2114,2016$.

[22] Zeroual Abdelhafid, Fouzi Harrou, and Ying Sun. Road traffic density estimation and congestion detection with a hybrid observer-based strategy. Sustainable Cities and Society, 46, 2019.

[23] Fouzi Harrou, Ying Sun, Muddu Madakyaru, and Benamar Bouyedou. An improved multivariate chart using partial least squares with continuous ranked probability score. IEEE Sensors Journal, 18(16):6715-6726, 2018.

[24] F. Harrou, L. Fillatre, and I. Nikiforov. Anomaly detection/detectability for a linear model with a bounded nuisance parameter. Annual Reviews in Control, 38(1):32-44, 2014.

[25] Fouzi Harrou, Lionel Fillatre, Michel Bobbia, and Igor Nikiforov. Statistical detection of abnormal ozone measurements based on constrained generalized likelihood ratio test. In 52nd IEEE Conference on Decision and Control, pages 4997-5002. IEEE, 2013.

[26] Fouzi Harrou, Abdelhafid Zeroual, and Ying Sun. Traffic congestion detection based on hybrid observer and glr test. In 2018 Annual American Control Conference (ACC), pages 604-609. IEEE, 2018.

[27] Xiaoxia Li, Qiang Yang, Zhuo Lou, and Wenjun Yan. Deep learning based module defect analysis for large-scale photovoltaic farms. IEEE Transactions on Energy Conversion, 34(1):520-529, 2018.

[28] Albert Yaw Appiah, Xinghua Zhang, Ben Beklisi Kwame Ayawli, and Frimpong Kyeremeh. Long short-term memory networks based automatic feature extraction for photovoltaic array fault diagnosis. IEEE Access, 7:30089-30101, 2019.

[29] Wei Zhou, Hongxing Yang, and Zhaohong Fang. A novel model for photovoltaic array performance prediction. Applied energy, 84(12):1187$1198,2007$.

[30] Fouzi Harrou, Bilal Taghezouit, and Ying Sun. Robust and flexible strategy for fault detection in grid-connected photovoltaic systems. Energy Conversion and Management, 180:1153-1166, 2019. 